\title{
Source of $C P$ Violation for Baryon Asymmetry of the Universe
}

\author{
Wei-Shu Hou ${ }^{1,2,3,4}$ \\ ${ }^{1}$ Department of Physics, ${ }^{2}$ Institute of Astrophysics, ${ }^{3}$ National Center for Theoretical Sciences, \\ ${ }^{4}$ Leung Center for Cosmology and Particle Astrophysics, \\ National Taiwan University, Taipei, Taiwan 10617
}

(Dated: October 30, 2018)

\begin{abstract}
The baryon asymmetry of the Universe requires $C P$ violation, but the Standard Model falls short by a factor of $10^{-10}$ or more. Starting from a clue at the $B$ factories, we point out that the large Yukawa couplings of the sequential fourth generation $t^{\prime}$ and $b^{\prime}$ quarks can provide enhancement by a factor of over $10^{13}$, making the 2-3-4 generation quark sector a viable source of $C P$ violation for the baryon asymmetry of the Universe. With recent hints of large $\sin 2 \Phi_{B_{s}}$ in $B_{s}^{0}-\bar{B}_{s}^{0}$ mixing from the Tevatron, the ultimate test would be to discover the $t^{\prime}$ and $b^{\prime}$ quarks at the Large Hadron Collider.
\end{abstract}

PACS numbers: 11.30.Er, 12.15.Ff, 13.25.Hw, 98.80.Bp

The Big Bang created matter and antimatter equally, but today we see only protons, neutrons and electrons in our Universe; the baryon asymmetry of the Universe (BAU) seems 100\%. Baryogenesis, the elimination of antimatter while leaving behind some matter, is one of the most fundamental problems.

One prerequisite for BAU is 1] the violation of chargeparity symmetry (CPV). Laboratory measurements of CPV so far all confirm the Kobayashi-Maskawa (KM) source [2] in the Standard Model (SM) [3]. But the KM mechanism is known to fall short of what is needed for BAU by over 10 orders of magnitude! While this definitely motivates continued search in the laboratories, the $10^{-10}$ factor may seem insurmountable. In this Letter we point out a possible enhancement without changing the SM dynamics in any essential way: a sequential fourth quark generation could bridge the $10^{-10}$ gap.

The baryon-to-photon ratio of our Universe became precisely known with WMAP data on the Cosmic Microwave Background Radiation (CMBR) [4],

$$
\frac{n_{\mathcal{B}}}{n_{\gamma}}=\left(5.1_{-0.2}^{+0.3}\right) \times 10^{-10} .
$$

For every baryon, there are $2 \times 10^{9}$ photons in the $2.7^{\circ} \mathrm{K}$ CMBR. We see no antibaryons, however, so

$$
\mathcal{A}_{\mathrm{BAU}} \equiv \frac{n_{\mathcal{B}}-n_{\overline{\mathcal{B}}}}{n_{\mathcal{B}}+n_{\overline{\mathcal{B}}}}=100 \%
$$

The mystery is not so much the elimination of antimatter, but why a tiny fraction of matter, Eq. (1), remains.

In 1967, Sakharov wrote down [1] the three conditions for generating BAU: i) baryon number violation; ii) $C$ and $C P$ violation; iii) deviation from thermal equilibrium. Sakharov was influenced by the experimental discovery [5] of $C P$ violation in the form of $K_{L}^{0} \rightarrow \pi^{+} \pi^{-}$ decay occurring at the $2 \times 10^{-3}$ level. In the early 1970s, before the first two fermion generations were even established, Kobayashi and Maskawa (KM) noticed [2] that, if one extends to a third quark generation, the weak interaction could have a unique source of $\mathrm{CPV}$ in the quark sector. This mechanism became part of SM.
It is remarkable that the SM carries all the ingredients to satisfy [6, 7] the Sakharov conditions. Although conserved at the classical level, baryon number is violated [8] by the triangle anomaly. Remarkably, the extreme suppression at zero temperature becomes unsuppressed [6] for temperature $T$ above electroweak energies of order $100 \mathrm{GeV}$. For the second condition, the weak interaction violates $C$ invariance, and the $\mathrm{KM}$ mechanism violates $C P$ invariance. For the third condition, the electroweak phase transition (EWPhT) could [ [] be strong enough to cause deviation from equilibrium. We will return to this last point in our discussions. Let us understand why the KM theory, which can explain all $C P$ asymmetries in the laboratories, is $10^{-10}$ too small for BAU.

The gauge coupling $g$ of a $W^{-}$boson to the $\bar{u}_{i} d_{j}$ quark pair is modulated by the quark mixing matrix element $V_{i j}$. KM showed [2] that the $2 \times 2$ matrix is orthogonal with no phase, but the $3 \times 3$ matrix $V$ is unitary, with a unique weak phase. Furthermore, in the three generation KM theory (which we call SM3), if any two like charged quarks are equal in mass, it effectively reduces to a two generation theory with no phase. CPV involves all three generations of quarks of both charges.

By invariance arguments, Jarlskog pointed out [9] that $\operatorname{Im} \operatorname{det}\left[m_{u} m_{u}^{\dagger}, m_{d} m_{d}^{\dagger}\right]$ can be used as the measure of $\mathrm{CPV}$. The general CPV invariant in SM3 is

$$
\begin{aligned}
J= & \left(m_{t}^{2}-m_{u}^{2}\right)\left(m_{t}^{2}-m_{c}^{2}\right)\left(m_{c}^{2}-m_{u}^{2}\right) \\
& \left(m_{b}^{2}-m_{d}^{2}\right)\left(m_{b}^{2}-m_{s}^{2}\right)\left(m_{s}^{2}-m_{d}^{2}\right) A,
\end{aligned}
$$

where $A$ is twice the area of any triangle formed from the unitarity condition $V^{\dagger} V=I$. The $d b$ element of $V V^{\dagger}$ is one such triangle [3] probed at the $B$ factories,

$$
V_{u d} V_{u b}^{*}+V_{c d} V_{c b}^{*}+V_{t d} V_{t b}^{*}=0,
$$

which is illustrated by the small triangle in Fig. 1. By the uniqueness of the CPV phase in SM3 [2], all possible analogues to Eq. (4) give the same area. For example, the rather squashed triangle $O-V_{u s} V_{u b}^{*} S$ in Fig. 1, corresponding to $V_{u s} V_{u b}^{*}+V_{c s} V_{c b}^{*}+V_{t s} V_{t b}^{*}=0$, is the same in area. From Eq. (3) we see that $J$ vanishes if $A=0$, or if any pair of like charged quarks are degenerate. 


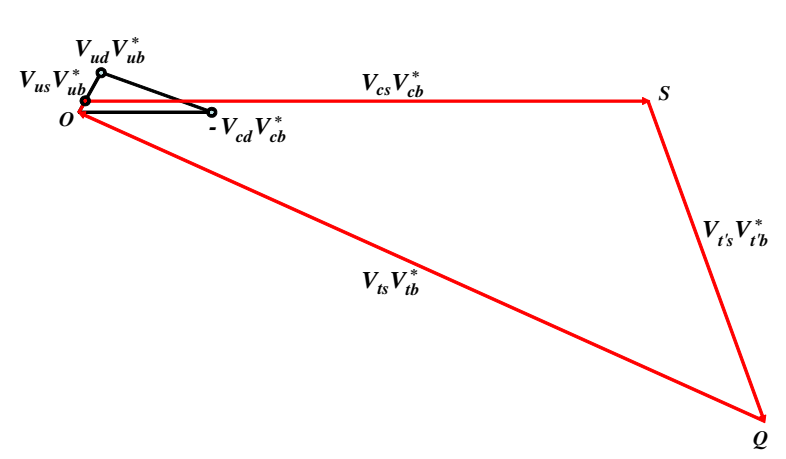

FIG. 1: Geometric representation of CPV in $b \rightarrow d$ and $b \rightarrow s$ transitions. The small triangle represents our knowledge of the three generation unitarity relation, Eq. (4). The large quadrangle represents the four generation relation, Eq. (7), drawn to scale with the $b \rightarrow d$ triangle, using $m_{t^{\prime}}=300 \mathrm{GeV}$ to account for Eq. (6), as well as consistency with $Z^{0}$ and kaon data. See text for details.

We can now see how CPV in SM3 falls far short of what is needed for baryogenesis. As $J$ has 12 mass dimensions, normalizing by the EWPhT temperature $T_{\mathrm{EW}} \sim 100$ $\mathrm{GeV}$, together with $A \simeq 3.1 \times 10^{-5}[3]$ one finds

$$
J / T_{\mathrm{EW}}^{12} \sim 10^{-20},
$$

which falls short of Eq. (1) by $10^{-10}$ or more [10].

SM3 can account for all CPV in the kaon and $B$ meson systems. In face of Eq. (5), many theories beyond SM3 that contain large enough CPV for BAU have therefore been proposed 11]. With recent advances in neutrino physics, the approach of generating BAU through the lepton sector, i.e. leptogenesis [12], has gained in popularity. We, however, aim to scrutinize the suppression in Eq. (3) further. Our clue is the recent " $\Delta \mathcal{A}_{K \pi}$ problem" revealed by the $\mathrm{B}$ factories.

In 2004, direct $\mathrm{CPV}$ in the decay of $B^{0}$ vs $\bar{B}^{0}$ mesons was established [13, 14], $\mathcal{A}_{K^{+} \pi^{-}} \equiv \mathcal{A}_{B^{0} \rightarrow K^{+} \pi^{-}} \cong-10 \%$. It is defined analogous to $\mathcal{A}_{\mathrm{BAU}}$, using the decay rates of $\bar{B}^{0} \rightarrow K^{-} \pi^{+}$vs $B^{0} \rightarrow K^{+} \pi^{-}$, and could still arise from SM3. The Belle experiment recently emphasized [15] a subtle, unexpected difference between charged and neutral $B$ mesons, $\Delta \mathcal{A}_{K \pi} \equiv \mathcal{A}_{K^{+} \pi^{0}}-\mathcal{A}_{K^{+} \pi^{-}}=+0.164 \pm$ 0.037. The world average 16 is now

$$
\Delta \mathcal{A}_{K \pi}=+0.147 \pm 0.027
$$

and well established. Although strong interaction effects cannot be ruled out, "it is equally possible that this is the first hint of an entirely new mechanism for particle-antiparticle asymmetry", through the so-called "electroweak penguin" process [17. Though apparently a far cry, does this offer new hope for BAU?

The difference $\Delta \mathcal{A}_{K \pi}$ is larger than the measured strength of $\mathcal{A}_{K^{+} \pi^{-}}$. What new physics CPV source could make such impact on the electroweak penguin amplitude $P_{\mathrm{EW}}$ ? The SM3 contribution to $P_{\mathrm{EW}}$ is dominated by

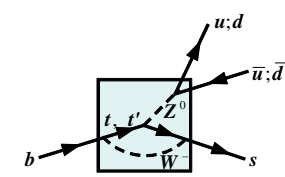

(a)

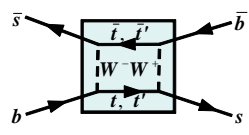

(b)
FIG. 2: The inner workings of (a) the electroweak penguin for $b \rightarrow s q \bar{q}$ transition, and (b) the box diagram for $\bar{s} b \leftrightarrow \bar{b} s$ transitions. Dashed lines are used for $W^{-}$and $Z^{0}$ bosons to indicate that the main effect comes from the longitudinal (or Goldstone boson) components.

the top quark, $P_{\mathrm{EW}}^{\mathrm{SM} 3} \propto V_{t s}^{*} V_{t b} f\left(m_{t}^{2}\right)$, which cannot affect $\Delta \mathcal{A}_{K \pi}$ because $V_{t s}^{*} V_{t b}$ carries no weak phase in SM3. Thus, new physics is called for if $P_{\mathrm{EW}}$ is the culprit. Note that the loop function $f\left(m_{t}^{2}\right)$ grows as $m_{t}^{2}$ to first approximation, rather than being suppressed by it: the top quark effect does not decouple from $P_{\mathrm{EW}}$ for large $m_{t}$. Utilizing this unusual nondecoupling behavior, we have advocated [18] that a natural possibility of generating $\Delta \mathcal{A}_{K \pi}$ is to add a top-like $t^{\prime}$ quark: the new quark mixing element product $V_{t^{\prime} s}^{*} V_{t^{\prime} b}$ carries a new CPV phase, and the impact on $P_{\mathrm{EW}}$ grows with $m_{t^{\prime}}^{2}$ ! This is still the KM theory, except one now has an extra, fourth quark generation. We call this SM4.

We illustrate the effect of $t$ and $t^{\prime}$ on $P_{\text {EW }}$ in Fig. 2(a). The $W$ boson loop around the $Z^{0}$ vertex converts the $b$ quark into an $s$ quark, and the $Z^{0}$ boson turns into a $\pi^{0}$. This is how $P_{\mathrm{EW}}$ enters $B^{\mp} \rightarrow K^{\mp} \pi^{ \pm}$. We have conducted analyses [18, 19] in SM4 using the state-of-theart perturbative QCD factorization approach, showing that the $t^{\prime}$ contribution can account for Eq. (6) through $P_{\text {EW }}$. Detailed checks were made [20] on $Z \rightarrow b \bar{b}$ decay and kaon data, finding that constraints [3] on $V_{i j}$ are satisfied. The outcome is illustrated in Fig. 1 for the nominal $m_{t^{\prime}}=300 \mathrm{GeV}$ (which is still consistent with Tevatron direct search bounds [3] ).

The triangles of SM3 become quadrangles in SM4. For $b \rightarrow s$ transitions the relevant quadrangle is

$$
V_{u s} V_{u b}^{*}+V_{c s} V_{c b}^{*}+V_{t s} V_{t b}^{*}+V_{t^{\prime} s} V_{t^{\prime} b}^{*}=0
$$

which is $O-V_{u s} V_{u b}^{*}-S-Q$ in Fig. 1. The strength of $V_{t^{\prime} s} V_{t^{\prime} b}^{*}$, i.e. the vector $\overrightarrow{S Q}$, which has a large angle with respect to $V_{c s} V_{c b}^{*}$ (the vector from $V_{u s} V_{u b}^{*}$ to $S$ which is practically real), is a consequence of Eq. (6). That is, the large phase and strength of $V_{t^{\prime} s} V_{t^{\prime} b}^{*}$, together with the nondecoupling of the $t^{\prime}$, generate the observed $\Delta \mathcal{A}_{K \pi}$. The vector $\overrightarrow{Q O}$, i.e. $V_{t s} V_{t b}^{*}$ in SM4, is very different from $\overrightarrow{S O}$ in SM3. Remarkably, the SM4 quadrangle of $V_{u d} V_{u b}^{*}+V_{c d} V_{c b}^{*}+V_{t d} V_{t b}^{*}+V_{t^{\prime} d} V_{t^{\prime} b}^{*}=0$ (not plotted), can barely be distinguished 20] from the small triangle of Eq. (4) for SM3. This explains why there was no indication of deviation from SM3 prior to Eq. (6). Note that for $m_{t^{\prime}}>300 \mathrm{GeV}$, the point $Q$ moves closer to $S$.

The quadrangle of Eq. (17) actually mimics a triangle, as $\left|V_{u s} V_{u b}^{*}\right|$ is very small. The area, an invariant measure 
of the strength of the $C P$ phase, is about 30 times the area $A$ of the SM3 triangle of Eq. (4). But this pales against the factor of $10^{10}$ needed for generating BAU, and one may despair. We observe, however, that the small size of $J$ in Eq. (3) is due less to the CP phase factor $A$, but rather to the powers of light quark masses, i.e. $m_{c}^{2} m_{b}^{4} m_{s}^{2} / T_{\mathrm{F} . \mathrm{N}}^{8} \sim 10^{-15}$. A similar point was mentioned by Peskin [17]. The masses of the $s$ and $c$ (and even $b$ !) quarks are tiny compared to the electroweak symmetry breaking scale of $v \simeq 246 \mathrm{GeV}$. In terms of Yukawa couplings $\lambda_{i}=\sqrt{2} m_{i} / v$, we have $\lambda_{s} \sim 0.0004, \lambda_{c} \sim 0.005$, and $\lambda_{b} \sim 0.017$.

Since it is the large Yukawa coupling, $\lambda_{t} \simeq 1$ for $m_{t} \simeq$ $170 \mathrm{GeV}$, that underlies the nondecoupling of the top in $P_{\mathrm{EW}}$, it may be the key to generating BAU as well. Shifting by one generation, one replaces Eq. (3) by

$$
\begin{aligned}
J_{(2,3,4)}^{s b} \simeq & \left(m_{t^{\prime}}^{2}-m_{c}^{2}\right)\left(m_{t^{\prime}}^{2}-m_{t}^{2}\right)\left(m_{t}^{2}-m_{c}^{2}\right) \\
& \left(m_{b^{\prime}}^{2}-m_{s}^{2}\right)\left(m_{b^{\prime}}^{2}-m_{b}^{2}\right)\left(m_{b}^{2}-m_{s}^{2}\right) A_{234}^{s b} \\
\sim & \frac{m_{t^{\prime}}^{2}}{m_{c}^{2}}\left(\frac{m_{t^{\prime}}^{2}}{m_{t}^{2}}-1\right) \frac{m_{b^{\prime}}^{4}}{m_{b}^{2} m_{s}^{2}} \frac{A_{234}^{s b}}{A} J
\end{aligned}
$$

in SM4, where $A_{234}^{s b} \simeq 10^{-3}$ is twice the area of the large triangle by shrinking $\left|V_{u s} V_{u b}^{*}\right| \rightarrow 0$ in Fig. 1 . The notation will be explained shortly. Taking $m_{t}<m_{b^{\prime}} \lesssim m_{t^{\prime}} \sim$ $300 \mathrm{GeV}$, one gains 15 orders of magnitude, with $10^{13}$ coming from Yukawa couplings (i.e. quark masses)! Using $m_{b^{\prime}} \sim m_{t^{\prime}} \sim 600 \mathrm{GeV}$, one gains another factor of $10^{2}$; the $\Delta \mathcal{A}_{K \pi}$ constraint on $\left|V_{t^{\prime} s} V_{t^{\prime} b}^{*}\right| m_{t^{\prime}}^{2}$ provides some control. In the context of BAU, the approximations made to obtain Eq. (8) are not so important compared to the striking gain by many orders of magnitude.

Eq. (8) is not just a guess. CPV can in general be written in terms of three-cycles, the trace of the cube of commutators of quark masses [21, 22]. With four generations, there are three [22] independent sources, one related to $J_{(2,3,4)}^{s b}$ (we have modified the more general notation of $J(2,3,4)$ of Jarlskog), another related to $J$, which could have been written as $J_{(1,2,3)}^{d b}$. However, compared to $v \simeq 246 \mathrm{GeV}, m_{d} \sim$ few $\mathrm{MeV}$ and $m_{s} \sim 100 \mathrm{MeV}$ are close to massless. In the $d-s$ degeneracy limit, the three sources reduce to a single one 22] (i.e. effectively 3 generation), which is nothing but Eq. (8). Indeed, $J$ is suppressed by the near degeneracy of $d-s-b$ as well as $u$-c, hence vanishingly small compared to $J_{(2,3,4)}^{s b}$, which is suppressed only by $m_{b}^{2}-m_{s}^{2}$.

There should be two extra CPV phases [3] compared to SM3. Does $J_{(2,3,4)}^{s b}$ capture the dominant effect for BAU in SM4? This is indeed the case. It is most easily seen by collapsing the $b \rightarrow s$ quadrangle in Fig. 1 to a triangle, by shrinking $V_{u s} V_{u b}^{*}$ to point $O$. The change in area is small, and the effective $2-4$ generation world again has a unique $C P$ phase, which is $A_{234}^{s b}$. Note that $J_{(2,3,4)}^{s b}$ by far dominates over $J$, unless $A_{234}^{s b} \ll A$ by $10^{-13}$. Thus, Eq. (8) gives the dominant effect of $C P$ violation relevant for BAU in SM4. Baryogenesis is possible with the dynamics that are already present in $S M$, and adding a fourth quark generation realizes it.

Some discussion is now in order.

First, our main result, the enhancement from replacing Eq. (3) by Eq. (8), does not depend on detailed values of $A_{234}^{s b}$, so long the latter does not vanish. Thus, the starting point of Eq. (6), and the subsequent discussion that lead to Eq. (8), are just scaffolding that can be removed once the observation is made.

Second, why has the prominent enhancement by the fourth generation gone unnoticed for so long? Since the $1990 \mathrm{~s}$, the fourth generation is perceived as ruled out [3] by electroweak precision measurements (EWPM) and $N_{\nu}$ counting. But we now know the neutrino sector is far richer than the naive SM, while the verdict from EWPM has been contested recently [23] (although $\left|m_{t^{\prime}}-m_{b^{\prime}}\right|$ mass splitting is indeed constrained). Our observation of a $10^{13}$ or more gain in CPV argues in strong favor of SM4 over SM3, since Eq. (5) shows that CPV in SM3 can never suffice for BAU. We remark that the enhancement does not work for vector-like exotic quarks, since their heaviness always lead to decoupling.

Third, most models with extra CPV for BAU tend to give too large EDMs (electric dipole moments; see Ref. 7] for some discussion). But for SM4, the same mechanisms that keep EDMs small in SM should be still at work.

Four, could a heavy fourth generation help bring about deviation from equilibrium as well? It is remarkable that EWPhT could in principle be strong enough in SM3 to satisfy Sakharov's third condition, but the current Higgs mass bound rules out [11] this possibility. It has therefore been popular 11] to introduce extra heavy bosons that couple strongly to the Higgs sector. There is recent speculation [24] regarding whether fermions that couple strongly to the Higgs sector could have similar effect. Though the top quark Yukawa coupling is not large enough, a model of higgsino and wino with large Yukawa couplings could strengthen the EWPhT into a first order one. A very recent study, however, stresses 25] that something similar is impossible for SM4 because of extra zero temperature corrections. These authors then resort to [26] $\tilde{t}^{\prime}$ and $\tilde{b}^{\prime}$ squarks, which is again falling back on the usual extra scalar boson approach.

In SUSY framework, one keeps all couplings perturbative, be it the Higgs self-coupling $\lambda$, or Yukawa couplings $\lambda_{i}$. However, we know that Nature does exploit strong, nonperturbative effects, e.g. in QCD. For Yukawa couplings corresponding to $m_{t^{\prime}}, m_{b^{\prime}}$ of order $600 \mathrm{GeV}$ or higher, unitarity violation sets in [27], and perturbation in Yukawa couplings breaks down. This does not necessarily mean that the theory ceases to exists, but rather, like in QCD, new bound states appear to restore unitarity. Such a picture has been advocated recently [28] for even the breaking of electroweak symmetry itself, without the need for a Higgs field (in other words, the Higgs becomes composite). If such is the case in Nature, the issue of strength of EWPhT should be revisited. This would depend on the actual high energy theory, but it would be remarkable if a heavy fourth generation could allow Stan- 
dard Model dynamics to account for baryogenesis. Such a picture, called electroweak baryogenesis [6, 7], would be one of the most beautiful outcomes of particle physics.

Five, our proposal offers exciting predictions that can be checked in the next few years at the Tevatron and the Large Hadron Collider (LHC). One prediction [18, 29] is large mixing-dependent $C P$ violation in the $B_{s}^{0}-\bar{B}_{s}^{0}$ system, in a measure called $\sin 2 \Phi_{B_{s}}$ that is akin to the established SM3 effect in the $B_{d}^{0}-\bar{B}_{d}^{0}$ system [3]. The $t$ and $t^{\prime}$ effect in the $\bar{s} b \leftrightarrow \bar{b} s$ box diagram is illustrated in Fig. 2(b). Like the $P_{\mathrm{EW}}$ amplitude of Fig. 2(a), these effects enjoy nondecoupling. The SM3 prediction of $\sin 2 \Phi_{B_{0}} \simeq-0.04$ is rather small [3] , but our prediction [18, 29] of $\sin 2 \Phi_{B_{s}} \sim-0.5$ to -0.7 for SM4 is rather striking. Recent reports from the Tevatron [30, 31] prefer the latter over SM. By a combined fit to these and various Tevatron results, the UTfit group [32] find a central value for $\Phi_{B_{s}} \sim-0.67$, though it may be too early to claim evidence. The result will certainly improve with more data. If $\sin 2 \Phi_{B_{s}}$ is large, it can be quickly measured to good precision by the $\mathrm{LHCb}$ experiment.

Note that a large and negative $\sin 2 \Phi_{B_{s}}$, though a consequence of $\Delta \mathcal{A}_{K \pi}>0$ in the four generation model, is not a requirement for Eq. (8) to be realized as the CPV source for BAU. Thus, the direct production of $t^{\prime}$ and $b^{\prime}$ quarks are of even more interest. The most recent Teva- tron bound on $m_{t^{\prime}}$ 33 is now approaching the $300 \mathrm{GeV}$ range. But whatever their masses, the $t^{\prime}$ and $b^{\prime}$ quarks can be readily discovered at the $\mathrm{LHC}$ ! We will learn in just a few years time whether Nature provides a fourth generation quark doublet with masses at several hundred $\mathrm{GeV}$, and with sufficient CPV for BAU.

Finally, we have not considered the associated 4 th generation heavy neutral and charged leptons, and the impact on neutrino physics. If a fourth generation of quarks is discovered at the LHC, the physics of the lepton sector would certainly be much richer.

In summary, noting that CPV in the three generation Standard Model is suppressed by the $s, c$ and $b$ quark masses hence too small for baryogenesis, we point out that, adding a fourth generation of quarks, one can gain a factor of $10^{13}$ or more. This could be the source of $C P$ violation for generating the baryon asymmetry of the Universe. Observation of large and negative $\sin 2 \Phi_{B_{s}}$ could offer further support, and the direct search for the fourth generation should be pursued vigorously at the Tevatron and the LHC to test this scenario.

Acknowledgement We thank F. Borzumati, T. Browder, P. Chang, L.-F. Li, E. Senaha, R. Sinha, A. Soni, C. Wagner, and R. Zwicky for discussions and advice.
[1] A.D. Sakharov, Pis'ma Zh. Eksp. Teor. Fiz. 5, 32 (1967) [JETP Lett. 5, 24 (1967)].

[2] M. Kobayashi and T. Maskawa, Prog. Theor. Phys. 49, 652 (1973).

[3] See W.M. Yao et al. (Particle Data Group), J. Phys. G 33, 1 (2006) for the current status.

[4] C.L. Bennett et al. (WMAP Collaboration), Astrophys. J. Suppl. 148, 1 (2003).

[5] J.H. Christenson, J.W. Cronin, V.L. Fitch, and R. Turlay, Phys. Rev. Lett. 13, 138 (1964).

[6] V.A. Kuzmin, V.A. Rubakov, and M.E. Shaposhnikov, Phys. Lett. B 155, 36 (1985).

[7] For a recent review, see the lectures by J.M. Cline, hep-ph/0609145, given at Les Houches, France, summer 2006.

[8] G. 't Hooft, Phys. Rev. Lett. 37, 8 (1976).

[9] C. Jarlskog, Phys. Rev. Lett. 55, 1039 (1985); Z. Phys. C 29, 491 (1985).

[10] M.B. Gavela et al., Nucl. Phys. B 430, 382 (1994); P. Huet and E. Sather, Phys. Rev. D 51, 379 (1995).

[11] See, for example, M. Dine and A. Kusenko, Rev. Mod. Phys. 76, 1 (2004).

[12] M. Fukugita and T. Yanagida, Phys. Lett. B 174, 45 (1986).

[13] Y. Chao et al. (Belle Collaboration), Phys. Rev. Lett. 93, 191802 (2004).

[14] B. Aubert et al. (BaBar Collaboration), Phys. Rev. Lett. 93, 131801 (2004).

[15] S.-W. Lin, Y. Unno, W.-S. Hou, P. Chang et al. (Belle Collaboration), Nature 452, 332 (2008).

[16] See the webpage of the Heavy Flavor Averaging Group (HFAG), http://www.slac.stanford.edu/xorg/hfag
[17] M.E. Peskin, Nature 452, 293 (2008).

[18] W.-S. Hou, M. Nagashima, and A. Soddu, Phys. Rev. Lett. 95, 141601 (2005).

[19] W.-S. Hou, H-n. Li, S. Mishima, and M. Nagashima, Phys. Rev. Lett. 98, 131801 (2007).

[20] W.-S. Hou, M. Nagashima, and A. Soddu, Phys. Rev. D 72, 115007 (2005).

[21] M. Gronau, A. Kfir and R. Loewy, Phys. Rev. Lett. 56, 1538 (1986).

[22] C. Jarlskog, Phys. Rev. D 36, 2128 (1987).

[23] G.D. Kribs, T. Plehn, M. Spannowsky, and T.M.P. Tait, Phys. Rev. D 76, 075016 (2007).

[24] M. Carena, A. Megevand, M. Quirós, and C.E.M. Wagner, Nucl. Phys. B 716, 319 (2005).

[25] R. Fok and G.D. Kribs, arXiv:0803.4207 [hep-ph].

[26] See also S.W. Ham, S.K. Oh, and D. Son, Phys. Rev. D 71, 015001 (2005).

[27] M.S. Chanowitz, M.A. Furman, and I. Hinchliffe, Phys. Lett. B 78, 285 (1978).

[28] B. Holdom, JHEP 0608, 076 (2006).

[29] W.-S. Hou, M. Nagashima, and A. Soddu, Phys. Rev. D 76, 016004 (2007).

[30] T. Aaltonen, et al. [CDF Collaboration], Phys. Rev. Lett. 100, 161802 (2008)

[31] V.M. Abazov et al. [D $\emptyset$ Collaboration], arXiv:0802.2255 [hep-ex].

[32] M. Bona et al. (UTfit Collaboration), arXiv:0803.0659 [hep-ph].

[33] See public note 9234 of the CDF Collaboration (http://www-cdf.fnal.gov/). 